\title{
Hydropriming para a promoção da emergência e do vigor em sementes provenientes de populações do bioma Caatinga
}

\author{
Hydropriming to promote emergence and vigor in seeds originating from groups derived from the \\ Caatinga biome
}

Hydropriming para promover la emergencia y el vigor en semillas provenientes de grupos derivados del bioma Caatinga

Recebido: 22/03/2021 | Revisado: 26/03/2021 | Aceito: 27/03/2021 | Publicado: 04/04/2021

\author{
Thiago Costa Ferreira \\ ORCID: https://orcid.org/0000-0002-2368-6223 \\ Instituto Nacional do Semiárido, Brasil \\ E-mail: thiago.ferreira@insa.gov.br \\ Manoel Rivelino Gomes de Oliveira \\ ORCID: https://orcid.org/0000-0003-1326-9749 \\ Universidade Federal da Bahia, Brasil \\ E-mail: manoel.oliveira@insa.gov.br \\ Aldrin Martin Perez-Marin \\ ORCID: https://orcid.org/ 0000-0001-9855-3284 \\ Instituto Nacional do Semiárido, Brasil \\ E-mail: aldrin.perez@insa.gov.br
}

\begin{abstract}
Resumo
O objetivo desta pesquisa foi testar o efeito do Hydropriming para a promoção da emergência e do vigor em sementes provenientes de populações do bioma Caatinga. Assim sendo, foram testadas as espécies: Sesbania virgata, Anadenanthera colubrina, Luetzelburgia auriculata, Cenostigma pyramidale, Vachelia farnesiana, Piptadenia retusa, Senna spectabilis, Mimosa tenuiflora, Sarcomphalus joazeiro e Enterolobium contortisiliquum. Houveram dois tratamentos: 1) imersão em água (Hydropriming) por $24 \mathrm{~h}$ a $25^{\circ} \mathrm{C}$ e 2) incubação por $24 \mathrm{~h}$ a $25^{\circ} \mathrm{C}$ sem imersão. Foram avaliadas variáveis de emergência e vigor. Os resultados obtidos foram testados por Qui-quadrado e testes Nãoparamétricos. A tecnologia de Hydropiming não foi vantajosa para espécies estudadas nesta pesquisa, exceto para Anadenanthera colubrina e Luetzelburgia auriculata. Sendo, portanto, a imersão por $24 \mathrm{~h}$ a $25^{\circ} \mathrm{C}$ potencial para promoção da emergência e vigor de A. colubrina e L. auriculata, outras pesquisas podem ser realizadas para melhorar a utilização desta metodologia em virtude de um melhor manejo destas espécies.
\end{abstract}

Palavras-chave: Tecnologia de sementes; Florestal; Inovação.

\begin{abstract}
The objective of this research was to test the effect of Hydropriming to promote the emergence and vigor in seeds of members of the Caatinga biome. Therefore, the following species were tested: Sesbania virgata, Anadenanthera colubrina, Luetzelburgia auriculata, Cenostigma pyramidale, Vachelia farnesiana, Piptadenia retusa, Senna spectabilis, Mimosa tenuiflora, Sarcomphalus joazeiro and Enterolobium contortisiliquum. There were two treatments: 1) immersion in water (Hydrocondditioning) for $24 \mathrm{~h}$ at $25^{\circ} \mathrm{C}$ and 2) incubation for $24 \mathrm{~h}$ at $25^{\circ} \mathrm{C}$ without immersion. Emergence and vigor variables were assessed. The results obtained were tested by Chi-square and nonparametric tests. Hydropiming technology was not advantageous for species studied in this research, except for Anadenanthera colubrina and Luetzelburgia auriculata. Therefore, a $24 \mathrm{~h}$ immersion at $25^{\circ} \mathrm{C}$ is potential to promote the emergence and vigor of Anadenanthera colubrina and Luetzelburgia auriculata, further research can be carried out to improve the management of these species.
\end{abstract}

Keywords: Seed Technology, Forestry, Inovation.

\section{Resumen}

El objetivo de esta investigación fue probar el efecto del hidrocondicionamiento para la promoción de la emergencia y el vigor en plántulas de semillas de áreas del bioma Caatinga. Así, se ensayaron como semillas de las especies: Sesbania virgata, Anadenanthera colubrina, Luetzelburgia auriculata, Cenostigma pyramidale, Vachelia farnesiana, Piptadenia retusa, Senna spectabilis, Mimosa tenuiflora, Sarcomphalus joazeiro y Enterolobium contortisiliquum. Estos fueron tratados en dos tratamientos diferentes: 1) inmersión en agua (Hydropriming) durante 24 ha $25^{\circ} \mathrm{C}$ y 2 ) incubación durante 24 ha $25^{\circ} \mathrm{C}$ sin inmersión. Se evaluaron variables de emergencia y vigor. Los resultados obtenidos se probaron mediante Chi-cuadrado y pruebas no paramétricas. La tecnología de hidrolizado no resultó 
ventajosa para las especies estudiadas en esta investigación, a excepción de Anadenanthera colubrina y Luetzelburgia auriculata. Por lo tanto, una inmersión de 24 horas a $25^{\circ} \mathrm{C}$ tiene el potencial de promover la emergencia y el vigor de Anadenanthera colubrina y Luetzelburgia auriculata, se pueden realizar más investigaciones para mejorar el manejo de estas especies.

Palabras clave: Tecnología de semillas; Silvicultura; Innovación.

\section{Introdução}

Pesquisas na área de tecnologia de sementes com respeito a espécies vegetais provenientes do bioma Caatinga são importantes para que se possa melhorar o conhecimento sobre as características do bioma, afim de otimizar ações de florestamento e, ainda, promover o uso sustentável nessa área (Mesquita, Dantas e Cairo, 2018; Dantas et al., 2019; Dantas et al., 2020; Oliveira et al., 2020). Tais pesquisas podem ser justificadas pelo fato que este bioma vem sofrendo muito com a ação antrópica, fator que tem diminuído populações vegetais no referido bioma, ocasionando a extinção de espécies e, assim, diminuindo o desenvolvimento regional (Oliveira et al., 2020).

Outrossim, as dinâmicas ecológicas visualizadas neste no bioma Caatinga são únicas e que as ações de reposição florestal descritas anteriormente necessitam de métodos peculiares e adaptados, haja vista os mecanismos de sobrevivência das espécies vegetais (Nascimento, Siqueira Filho \& Meiado, 2018; Lima \& Meiado, 2019) principalmente com respeito as sementes em relação a necessidade de proteção contra a variação da umidade no substrato (Lima, Oliveira \& Meiado, 2018; Nascimento, Siqueira Filho \& Meiado, 2018; Lima \& Meiado, 2019), as altas temperaturas (Mesquita, Dantas \& Cairo, 2018) e a presença de áreas com índices de salinidade (Dantas et al., 2019; Dantas et al., 2020), por exemplo.

$\mathrm{O}$ tratamento de sementes com uso de primings tem sido um dos mais recomendados para otimizar o manejo sustentável de espécies florestais no mundo, promovendo assim, em linhas gerais, a melhoria da qualidade da germinação e vigor, que influenciam na sanidade e fisiologia de plântulas frente a situações de estresse (Rifna et al., 2019), exemplificadas no parágrafo anterior. Xue et al. (2018) e Rifna et al. (2019) descrevem exemplos positivos da utilização de técnicas de tratamento de sementes em relação a melhoria da qualidade da emergência e vigor em essências florestais, inclusive com populações vegetais provenientes de áreas com características semelhantes ao bioma Caatinga.

O hydropriming, como exemplo, segundo Rifna et al. (2019), é uma das técnicas mais simplificadas que pode também consistir simplesmente na imersão em água de sementes por um dado período e temperatura, antes da realização do semeio. Este processo apresenta baixo custo e facilidade de realização, segundo afirmam Pereira (2011) e Rocha et al. (2018). Algumas espécies provenientes da Caatinga já foram estudadas quanto a utilização da referida tecnologia com adaptações, podendo serem destacados os seguintes exemplos Garcinia gardneriana (Rocha et al., 20218), Mimosa tenuiflora (Lima \& Meiado, 2019), Pilosocereus catingicola subsp. salvadorensis (Lima \& Meiado, 2018).

Ligando os pontos conceituais apresentados anteriormente, pode ser possível a testagem dos efeitos da técnica de Hydropriming, com a testagem desta tecnologia em relação as pouco estudadas espécies da Caatinga (Mesquita, Dantas \& Cairo, 2018; Dantas et al., 2019; Dantas et al., 2020; Oliveira et al., 2020). Dentre estas espécies, foram destacadas nesta pesquisa Sesbania virgata, Anadenanthera colubrina, Luetzelburgia auriculata, Cenostigma pyramidale, Vachelia farnesiana, Piptadenia retusa, Senna spectabilis, Mimosa tenuiflora, Sarcomphalus joazeiro e Enterolobium contortisiliquum para a experimentação. A seguir, serão detalhadas algumas informações sobre cada uma destas espécies.

Sesbania virgata (Cav.) Poir. [sin.: Sesbania marginata Benth.] é uma espécie nativa do Brasil, com ocorrência em ambientes semiárido e com estações secas. Suas sementes apresentam indícios de dormência (Araújo et al., 2004). Masetto et al. (2013) descrevem esta espécie como pioneira e com grande afinidade com bactérias que são nitrificantes. 
Anadenanthera colubrina (Vell.) Brenan [sin.: Piptadenia colubrina (Vell). Benth.] é uma_espécie ocorrente no Brasil, com ampla distribuição, utilizada para a produção de madeira, medicamentos e forragem. Suas sementes são frágeis e delgadas e não apresentam dormência (Bispo, 2017).

Luetzelburgia auriculata (Allemão) Ducke [sin.: Bowdichia freirei Ducke] (Fabaceae) é uma espécie endêmica do Brasil, com sementes que apresentam tegumento endurecido. Também é descrita como sendo portadora de propriedades medicinais e que estudos sobre sua ecologia ainda são restritos na literatura (Nogueira et al., 2012)

Cenostigma pyramidale (Tul.) Gagnon \& G.P. Lewis [sin.:Poincianella pyramidalis (Tul.) L.P. Queiroz] (Fabaceae) é uma árvore endêmica do Brasil, com ampla disseminação no bioma Caatinga e com usos diversos, principalmente forragem e madeira. Também é relatada como pioneira e uma importante espécie na sucessão ecológica (Mattias et al., 2018).

Enterolobium contortisiliquum (Vell.) Morong [sin.: Mimosa contortisiliqua Vellozo] (Fabaceae) é uma espécie arbórea, terrícola e com presença em vários biomas brasileiros. Suas sementes são duras protegidas por uma frutificação lignificada (Flora do Brasil, 2021).

Vachelia farnesiana (L.) Wight \& Arn. [sin.: Acacia farnesiana (L.) Willd.] (Fabaceae) é uma espécie arbustiva, perene, usada como pasto apícola e como forrageira para o gado. Apresenta também a característica de ser pioneira e suas sementes apresentam grande durabilidade (Moraes et al., 2012).

Piptadenia retusa P.G. Ribeiro, Seigler \& Ebinger [sin.: Mimosa retusa Jacq.] (Fabaceae) é uma espécie arbustiva, encontrada em áreas de Caatinga, pioneira e que é apresentada como útil para o pasto apícola e forragem. Suas sementes apresentam uma dormência tegumentar e longevidade apuradas (Paula et al., 2016).

Senna spectabilis (DC.) H.S.Irwin \& Barneby [sin.: Cassia spectabilis DC.] (Fabaceae) é uma espécie terrícola, arbustiva e com grande disseminação em todo o país. Em geral, suas sementes são portadoras de dormência fisiológica e tegumentar, fator que diminui a sua germinação efetivamente (Jeller et al., 2001; Lima et al., 2018).

Mimosa tenuiflora (Willd.) Poir. [sin.: Acacia tenuiflora Willd.] (Fabaceae) é uma espécie ocorrente e bastante disseminada em áreas de Caatinga que apresenta sementes pequenas e com resistência tegumentar acentuada, segundo descrevem Santos et al. (2018). Pois na qual pode ser usada a ação de ácidos para melhorar a uniformidade e agilizar a germinação (Brasil, 2009).

Sarcomphalus joazeiro (Mart.) Hauenshild Mart. [sin.: Ziziphus joazeiro Mart.] (Rhamaceae) é uma espécie vegetal presente no semiárido brasileiro. Suas sementes são encontradas em frutos mucilaginosos e com copa globosa, existe uma dormência acentuada em suas sementes. Utilizada na medicina popular, alimentícia (frutos) e pasto (Andrade et al., 2019; Bispo et al., 2019; Gomes, 2019).

Sendo assim, este trabalho tem como base a experimentação da tecnologia de Hydropriming para a promoção da emergência em sementes de dez espécies ocorrentes na Caatinga (Sesbania virgata, Anadenanthera colubrina, Luetzelburgia auriculata, Cenostigma pyramidale, Vachelia farnesiana, Piptadenia retusa, Senna spectabilis, Mimosa tenuiflora, Sarcomphalus joazeiro e Enterolobium contortisiliquum).

\section{Metodologia}

\subsection{Espécies}

Para a realização desta pesquisa foram utilizadas sementes providas pelo NEMA (Núcleo de Ecologia e Monitoramento Ambiental) ligado à UNIVASF (Universidade Federal do Vale do São Francisco), de acordo com as proposições e metodologias de acompanhamento e monitorização ecológica do entorno da obra da Transposição do Rio São Francisco. A metodologia de coleta e a área geográfica amostral, bem como outras informações sobre os trabalhos do referido núcleo, podem ser visualizadas no sítio <https://www.nema.univasf.edu.br/site/>, acesso em 26.02.2021. As espécies utilizadas 
foram: Sesbania virgata, Anadenanthera colubrina, Luetzelburgia auriculata, Cenostigma pyramidale, Vachelia farnesiana, Piptadenia retusa, Senna spectabilis, Mimosa tenuiflora, Sarcomphalus joazeiro e Enterolobium contortisiliquum, referentes ao pedido número 593.

\subsection{Hydropriming e delineamento experimental}

Os ensaios foram montados concomitantemente, entre os meses de janeiro e fevereiro de 2021 e eram independentes entre si. As sementes de todas as espécies citadas foram divididas em dois grupos, de cem sementes cada (quatro repetições de 25 sementes cada), conforme Pádua et al. (2020). Cada grupo foi depositado separadamente em copos de polietileno de 200mL, um lote tratado com o Hydropiming (imersão em água destilada) e outro lote não foi imerso em nenhuma substância, ambos os grupos foram acondicionados por $24 \mathrm{~h}$ na temperatura de $25 \pm 2{ }^{\circ} \mathrm{C}$ e luminosidade natural por cerca de $12 \mathrm{~h}$. Após este período, as sementes foram semeadas em canteiro com solo (Tabela 1).

Tabela 1. Análise física do substrato (solo) utilizado para o semeio $(0-20 \mathrm{~cm}$ de profundidade).

\begin{tabular}{|c|c|c|c|c|c|}
\hline pH & $\mathbf{P}$ & $\mathbf{K}^{+}$ & $\mathrm{Na}^{+}$ & $\mathbf{H}^{+}+\mathbf{A l}^{+3}$ & $\mathbf{A l}^{+3}$ \\
\hline $\mathrm{H}_{2} \mathrm{O}(1: 2,5)$ & \multicolumn{2}{|c|}{$\mathrm{mg} / \mathrm{dm}^{3}$} & \multicolumn{3}{|c|}{------------- cmolc/dm³ ------- } \\
\hline 5,4 & 3,3 & 98,09 & 0,11 & 3,37 & 0,2 \\
\hline $\mathbf{C a}^{+2}$ & $\mathrm{Mg}^{+2}$ & $\mathrm{~V} \%$ & CTC & \multicolumn{2}{|c|}{ MO } \\
\hline & ------- & $n^{3}----$ & & \multicolumn{2}{|c|}{$\mathrm{g} / \mathrm{kg}$} \\
\hline 3,34 & 0,32 & 54,4 & 7,39 & \multicolumn{2}{|c|}{6,46} \\
\hline
\end{tabular}

Legenda: P, K, Na: Extração por Mehlich; H + Al: Extrator Calcio acetato 0,5 M, pH 7,0; pH 7,0; Al, Ca, Mg: Extrator KCl 1 M; MO.: Matéria orgânica - Walkley-Black; V\%. Fonte: Laboratório de Solos (UFCG).

\subsection{Método analítico e estatístico}

Foram avaliadas a percentagem de emergência (PC) em uma primeira contagem (com sete dias após o semeio) e em uma contagem final (E) (vinte e cinco dias após o semeio), conforme descrito na RAS (Regra de Análise de Sementes) (Brasil, 2009), percentagem de plântulas mortas ou sementes não germinadas (NG) e o índice de velocidade de germinação (IVE), segundo descrevem Ferreira et al. (2021). As contagens de plântulas emergidas foram realizadas até que o estande fosse estabilizado. Nenhuma das sementes trabalhadas nesta pesquisa recebeu tratamento para a quebra da dormência.

Os tratamentos foram analisados com o uso da análise de Qui-quadrado $\left(X^{2}\right)$, a 5,00\% de significância, o teste foi realizado a um nível de significância de 5\%, e neste caso rejeita-se $\mathrm{H}_{0}$ quando $\mathrm{p}$ - valor $\leq 0,05$ e aceita $\mathrm{H}_{0}$ caso contrário. Também houve a testagem dos dados com o uso do teste de Shapiro-Wilk. As médias obtidas foram tratadas com a utilização da estatística descritiva (média por tratamento, média geral, erro e desvio padrões). Foram realizados os cálculos estatísticos descritos para todas as espécies que obtiveram uma emergência mínima de 20,00\% no estande final em pelo menos um dos tratamentos realizados. Caso não fosse alcançada essa meta, somente foi realizada a estatística não paramétrica dos dados, a fim de melhor apresentá-los nesta pesquisa.

\section{Resultados e Discussão}

\subsection{Teste de Shapiro-Wilk}

Para todas as variáveis em todos os ensaios que foram realizados o teste de Qui-quadrado foram constatadas normalidade entre os dados obtidos, exceto para as variáveis G e NG em relação a espécie Enterolobium contortisiliquum (Tabela 1). 
Tabela 1. Teste de Shapiro-Wilk para os resultados das variáveis percentagem de primeira contagem de emergência (PC), emergência final (E), percentagem de plântulas mortas ou sementes não germinadas (NG) e o índice de velocidade de germinação (IVE) em relação aos dados obtidos nos tratamentos de sementes (ausência e presença de Hydropriming) em sementes de Sesbania virgata, Anadenanthera colubrina, Luetzelburgia auriculata, Cenostigma pyramidale e Enterolobium contortisiliquum.

\begin{tabular}{lllll}
\hline \multirow{2}{*}{ ESPÉCIE } & \multicolumn{2}{l}{ Teste de Shapiro-Wilk } & NG & IVE \\
\cline { 2 - 5 } & PC & E & 0,0741 & 0,0741 \\
\hline Sesbania virgata & 0,1632 & 0,6423 & 0,6423 & 0,0854 \\
Anadenanthera colubrina & 0,592 & 0,5096 & 0,5096 & 0,947 \\
Luetzelburgia auriculata & 0,5224 & 0,2024 & 0,2024 & 0,1417 \\
Cenostigma pyramidale & 0,893 & 0,0199 & 0,0199 & 0,1184 \\
Enterolobium contortisiliquum & 0,0816 & & \\
\hline
\end{tabular}

Fonte: Autores.

\subsection{Sesbania virgata}

Em relação aos resultados obtidos para a espécie Sesbania virgata pode ser descrito que não houve diferença estatística para os tratamentos analisadas em relação a variável IVE ( $p$ - valor: 0,8221). Foram significativas as diferenças entre os tratamentos nas variáveis PC ( $p$ - valor: 0,1119), E ( $p$ - valor: 0,1158) e NG ( $p$ - valor: 0,10130).

As médias obtidas para o tratamento sem imersão foram superiores aos relatados para as médias do tratamento com imersão em cerca de 30,90 e 23,07 \% para as variáveis PC e E, respectivamente; já para as variáveis cerca de 41,17 e 47,59\% abaixo do tratamento com imersão para as variáveis NG e IVE, respectivamente (Tabela 3).

Em relação à variável PC, os resultados para o tratamento sem inoculação estão próximos aos descritos por Araújo et al. (2004). Em relação aos resultados obtidos para a variável E pode ser descrito que os valores se assemelham aos descritos por Araújo et al. (2004) e por Silva et al. (2011), porém vale ser descrito que em ambos os artigos citados houveram tratamentos para a quebra da dormência das sementes, não sendo utilizada nesta pesquisa.

Provavelmente, a origem da população utilizada nesta pesquisa apresenta a característica de emergir mais uniformemente em resposta ao estímulo ambiental favorável. Fator que pode ser também visualizado nos escritos de Azeredo et al. (2010), Azeredo et al (2011), Benedito et al. (2011) quando trabalharam com populações diferenciadas de Pityrocarpa moniliformis, uma outra Fabaceae encontrada no bioma Caatinga, semelhante em alguns aspectos botânicos a S. virgata (Flora do Brasil, 2021).

Outrossim, houve uma diminuição do estande entre a primeira contagem e a data da contagem da emergência (final do ensaio), fator visualizado indiretamente na variável IVE pelo valor diminuto visualizado em relação ao tratamento sem imersão (Tabela 3).

Nesse sentido, pode ter ocorrido alguma ação bioquímica que promoveu a germinação de plântulas anormais e estas vieram a sucumbir por ação dos agentes que circundam a semente, normais segundo os escritos de Rifna e colaboradores (2019). A mortalidade ou não germinação foi maior no tratamento com imersão, semelhante às informações descritas por Oliveira et al. (2018) quando trabalharam com a imersão de sementes de Jacaranda brasiliana, outra espécie encontrada na Caatinga. Sementes de S. virgata não foram positivas a utilização da imersão, provavelmente devido a sua natureza pioneira, que permite que sementes germinam em condições ambientais inóspitas. Também por sua condição em relação a dessecação sendo assim, provavelmente, necessária apenas uma pequena quantidade de água para o iniciar e prosseguir das reações da germinação, segundo as informações descritas por Araújo et al. (2004) e por Silva et al. (2011). 
Tabela 2. Estatística descritiva para os resultados das variáveis percentagem de primeira contagem de emergência (PC), emergência final (E), percentagem de plântulas mortas ou sementes não germinadas (NG) e o índice de velocidade de germinação (IVE) em relação aos dados obtidos nos tratamentos de sementes (ausência e presença de Hydropriming) em sementes de Sesbania virgata, Anadenanthera colubrina, Luetzelburgia auriculata, Cenostigma pyramidale e Enterolobium contortisiliquum.

\begin{tabular}{llllll}
\hline & & MÉDIAS & & \\
\hline ESPÉCIE & TRATAMENTO & PC & G & NG & IVE \\
\hline \multirow{2}{*}{ Sesbania virgata } & Imerso & 17,00 & 15,00 & 85,00 & 2,70 \\
Média geral & Sem imersão & 55,00 & 65,00 & 35,00 & 5,68 \\
Erro padrão & & 36,00 & 40,00 & 60,00 & 6,34 \\
Desvio padrão & & 7,48 & 9,94 & 9,94 & 1,43 \\
\hline \multirow{2}{*}{ Anadenanthera colubrina } & Imerso & 21,17 & 28,12 & 28,12 & 4,03 \\
Média geral & Sem imersão & 46,00 & 84,00 & 16,00 & 9,07 \\
Erro padrão & & 39,00 & 55,00 & 45,00 & 7,67 \\
Desvio padrão & & 3,00 & 69,50 & 30,50 & 8,37 \\
\hline \multirow{2}{*}{ Luetzelburgia auriculata } & & 1,81 & 6,14 & 6,14 & 0,45 \\
Média geral & Imerso & 4,13 & 17,36 & 17,36 & 1,27 \\
Erro padrão & Sem imersão & 8,00 & 51,00 & 49,00 & 3,41 \\
Desvio padrão & & 6,00 & 43,00 & 57,00 & 3,14 \\
\hline \multirow{2}{*}{ Cenostigma pyramidale } & & 1,31 & 5,00 & 53,00 & 3,28 \\
Média geral & Imerso & 6,70 & 14,14 & 5,00 & 0,29 \\
Erro padrão & Sem imersão & 79,00 & 72,00 & 28,00 & 11,95 \\
Desvio padrão & & 73,50 & 72,00 & 18,00 & 13,56 \\
\hline \multirow{2}{*}{ Enterolobium contortisiliquum } & & 3,46 & 2,59 & 23,00 & 12,75 \\
Média geral & Imerso & 14,00 & 13,00 & 87,00 & 2,22 \\
Erro padrão & Sem imersão & 20,00 & 22,00 & 78,00 & 3,52 \\
Desvio padrão & & 17,00 & 17,50 & 82,50 & 2,87 \\
& & 1,81 & 2,38 & 2,38 & 0,33 \\
\hline
\end{tabular}

Fonte: Autores.

\subsection{Anadenanthera colubrina}

Em relação aos resultados obtidos para a espécie Anadenanthera colubrina pode ser descrito que não houve diferença estatística entre os tratamentos para as variáveis PC ( $p$ - valor: 0,9173), E (p - valor: 0,3865) e IVE (p - valor: 0,9513). Foram significativas as diferenças na variável NG ( $\mathrm{p}$ - valor: 0,0008).

As médias obtidas para o tratamento sem imersão foram superiores aos relatados para as médias do tratamento com imersão em cerca de 14,28 e 18,20\% para as variáveis PC e IVE, respectivamente; para a variável E a diferença foi de 52,72\% acima do tratamento sem imersão e para a variável NG o tratamento com imersão promoveu um valor de 35,55\% abaixo do tratamento sem imersão (Tabela 3). Os resultados descritos para as variáveis PC e E são semelhantes aos demonstrados por Silva et al. (2019) e Cruz et al. (2020).

O tratamento de imersão promoveu uma quantidade menor de sementes não germinadas e plântulas mortas e ainda promoveu um IVE maior. Fatores que configuram um resultado positivo para a utilização desta técnica para melhorar o manejo dessa espécie (Tabela 3). Porém, as sementes de A. colubrina são delgadas, como descrevem Silva et al. (2019) e Cruz et al. (2020), provavelmente seria este fato que a técnica do Hydropriming foi eficaz (Rifna et al. 2019), mas esse fator pode ser prejudicial se as sementes serem retiradas do contato com o tratamento não foram cuidadosamente transferidas e semeadas com rapidez e sem fazer pressão sobre estas. Pois, segundo Castro et al. (2017), as sementes de A. colubrina após o período de 
24h de imersão iniciam a sua produção de tecidos, referentes a fase dois da germinação, estando pouco tenras em comparação com uma semente não imersa e assim susceptíveis a danos mecânicos.

Outrossim, este teste foi realizado irrigado com água de chuva tratada e os padrões condutibilidade elétrica poderam ter 7iminuído a germinação de sementes de A. colubrina, conforme descrevem Rego et al. (2007) que estudaram o efeito da salinidade na germinação de sementes desta espécie. Também Oliveira et al. (2012) descrevem que substratos com um balanço entre as argilas e areias que compõem o substrato (Tabela 1), podem ser efetivos para melhorar a germinação de sementes $A$. colubrina. No caso do substrato utilizado na pesquisa e descrito na Tabela 1, houve, provavelmente, uma facilitação da emergência das sementes trabalhadas nesta pesquisa.

Maiores informações sobre a germinação de sementes de A. colubrina com respeito a luz, substratos, salinidade e déficit hídrico podem ser vistas nos escritos de Santos et al. (2007) e Oliveira et al. (2012), fatores descritos por esses autores provavelmente interferiram na emergência destas sementes.

\subsection{Luetzelburgia auriculata}

Em relação aos resultados obtidos para a espécie Luetzelburgia auriculata pode ser descrito que não houve diferença estatística nas variáveis PC ( $\mathrm{p}$ - valor: 0,05756) e IVE ( $\mathrm{p}$ - valor: 0,8933). Foram significativas as diferenças entre os tratamentos nas variáveis $\mathrm{E}$ ( $\mathrm{p}$ - valor: 0,0013) e NG ( $\mathrm{p}$ - valor: 0,0089).

As médias obtidas para o tratamento sem imersão foi superior ao com imersão em 50,00\% para a variável PC. Cerca de 15,68 e 8,61\% foi o acréscimo que o resultado para o tratamento com imersão teve em relação ao tratamento sem imersão, para as variáveis E e NG, respectivamente. E ainda o tratamento com imersão foi cerca de $14 \%$ menor que o outro tratamento para a variável NG (Tabela 3).

Artigos que relatam dados sobre a emergência em sementes de L. auriculata são bem escassos na literatura, segundo afirmam Nogueira et al. (2012). Todas as variáveis analisadas nesta pesquisa, para os dois tratamentos experimentados, obtiveram resultados que são superiores aos descritos por Nogueira et al. (2012). Provavelmente, fatores fenotípicos, comuns em espécies de Fabaceae provenientes de áreas de Caatinga, podem ter sido a causa desta diferença, conforme afirmam Azeredo et al. (2010), Azeredo et al (2011) e Benedito et al. (2011).

\subsection{Cenostigma pyramidale}

Em relação aos resultados obtidos para a espécie $C$. pyramidale pode ser descrito que não houve diferença estatística em todas as variáveis PC ( $\mathrm{p}$ - valor: 0,2245), E ( $\mathrm{p}$ - valor: 0,9315$), \mathrm{NG}$ ( $\mathrm{p}$ - valor: 0,3940$)$ e IVE ( $\mathrm{p}$ - valor: 0,9977$)$. As médias obtidas para o tratamento sem imersão foram superiores ao tratamento com imersão em 13,92, 13,88 e 13,44\% para as variáveis PC, E e IVE e ainda foi cerca de 55,55\% menor para a variável NG (Tabela 3). Os resultados obtidos em todas as variáveis analisadas são semelhantes aos descritos por Matias et al. (2018) e Freitas et al. (2019).

Geralmente, são descritas populações da espécie $C$. pyramidale como sendo pioneiras, até mesmo em áreas antropizadas ou com déficit hídrico, provavelmente esta espécie não suporta bem a imersão de suas sementes por este aspecto (Mattias et al., 2018). A imersão de sementes por 24h também não foi eficiente para promover a germinação de sementes de Tachigali vulgaris, uma Fabaceae encontrada no Brasil, segundo descrevem Abreu, Porto e Nogueira (2017).

\subsection{Enterolobium contortisiliquum}

Em relação às variáveis analisadas não houveram diferenças significativas entre os tratamentos conforme o teste de $X^{2}$. Os valores de cada variância foram PC ( $p$ - valor: 0,6004$), E$ ( $p$ - valor: 0,5402$), N G$ ( $p$ - valor: 0,8690$)$ e IVE ( $p$ - valor: 
0,9982). Para esta espécie os valores obtidos para o tratamento sem imersão em relação às variáveis PC, E e IVE foram cerca de 30,00, 4,90 e 36,75\% superiores ao tratamento com imersão, respectivamente (Tabela 3).

Percentuais diminutos de germinação em sementes de Enterolobium contortisiliquum são esperadas em condições naturais e sem o uso de ácido para a quebra da dormência de acordo com os escritos de Sclaron et al. (2005), Trindade-Lessa et al (2015) e Lozano et al. (2016). Segundo estes autores, a constituição morfológica e bioquímicas das sementes de $E$. contortisiliquum contribuem para uma longevidade e integridade das sementes por longos períodos. Semelhante aos resultados descritos neste segmento, Rifna et al. (2019) descrevem diversas espécies florestais que não foram afetadas positivamente com a utilização de hydropriming antes do semeio.

\subsection{Vachellia farnesiana, Piptadenia retusa, Senna spectabilis, Mimosa tenuiflora e Sarcomphalus joazeiro}

As espécies reunidas neste segmento apresentaram menos de $20 \%$ de emergência em ambos os tratamentos. A seguir, foram descritos os percentuais e as possíveis correlações com informações descritas na literatura que possibilitam uma explicação dos fenômenos ocorrentes.

A média de emergência para Vachellia farnesiana foi de 8,00 e 12,00\% para os tratamentos com imersão e sem imersão. Moraes et al. (2012) afirmam que as sementes de V. farnesiana apresentam uma durabilidade acentuada em condições de campo, além de um tegumento endurecido. Segundo este mesmo grupo de autores, existe a necessidade de escarificação por ácido sulfúrico a 5\%, por 5 minutos ou a escarificação mecânica por meio de lixa $\mathrm{N}^{\circ} 08$ na região oposta ao hilo para que seja efetiva a germinação desta espécie, em até quinze dias após o plantio.

A média de emergência para Piptadenia retusa foi de 15,00 e 10,00\% para os tratamentos com imersão e sem imersão. Vários autores descrevem que sementes de Piptadenia spp. apresentam uma resistência tegumentar apurada e que a escarificação com ácido seria uma boa opção para a melhoria da germinação (Pessoa et al., 2010; Azevedo et al. 2010; Azevedo et al., 2016). Este fato poderia estar relacionado com a possibilidade de germinação desta espécie em virtude de um espaço temporal mais dilatado e com em condições ambientais que podem ser variantes quanto se trata de áreas no bioma Caatinga, segundo descrevem escritos de Azeredo et al. (2010), Azeredo et al (2011), Benedito et al. (2011) quando trabalharam com populações diferenciadas de Pityrocarpa moniliformis.

A média de emergência para Senna spectabilis foi de 12,00 e 11,00\% para os tratamentos com imersão e sem imersão. Sementes de S. spectabilis apresentam dormência fisiológica e tegumentar (Jeller et al., 2001; Lima et al., 2018). Inclusive, Lima e colaboradores (2018) descrevem que ciclos de hidratação e desidratação podem ser bem úteis para a germinação de sementes desta espécie. Santos et al. (2018) descrevem que existe uma resistência tegumentar acentuada e que a ação da hidratação descontínua sobre estas sementes pode ser uma importante alternativa para ser utilizada no lugar do tratamento ácido recomendado pela RAS (Brasil, 2009). Correia et al (2017) e Pereira et al. (2017) descrevem que sementes provenientes de áreas do bioma Caatinga apresentam resistência natural às intempéries ambientais, provavelmente o fator que ponderou o resultado descrito.

A média de emergência para Sarcomphalus joazeiro foi de 5,00 e 3,00\% para os tratamentos com imersão e sem imersão. Lucena et al. (2017) descrevem que sementes de juazeiro apresentam um dilatado período de germinação, fato comprovado na RAS que determina a visualização da germinação em uma única contagem aos 100 dias de semeio (Brasil, 2009). Provavelmente as condições adaptativas das populações vegetais encontradas no bioma Caatinga podem ser uma das explicações para um tão alargado período de germinação (Lima, Oliveira e Meiado, 2018; Nascimento, Siqueira Filho e Meiado, 2018; Lima e Meiado, 2019).

Em relação aos resultados descritos para as espécies Vachellia farnesiana, Piptadenia retusa, Senna spectabilis, Mimosa tenuiflora e Sarcomphalus joazeiro os resultados são muito importantes para uma melhor compreensão sobre as 
particularidades do bioma Caatinga (Nascimento, Siqueira Filho \& Meiado, 2018; Lima \& Meiado, 2019). Bioma único em peculiaridades ecológicas (Lima, Oliveira \& Meiado, 2018; Nascimento, Siqueira Filho e Meiado, 2018; Lima \& Meiado, 2019), ainda pouco estudado (Mesquita, Dantas \& Cairo, 2018) e com uma alta ação antrópica (Dantas et al., 2019). Tais dados servem para questionar que tecnologias podem não ser adaptadas ao manejo de espécies florestais da Caatinga (Dantas et al., 2020; Oliveira et al., 2020).

Tecnologias inovadoras ainda podem ser pesquisadas em relação aos fatores de hidratação e desidratação das sementes da Caatinga (Mesquita, Dantas \& Cairo, 2018; Dantas et al., 2019; Dantas et al., 2020; Oliveira et al., 2020), permitindo também a utilização de primings diversos baseados em hydropriming (Rifna et al., 2019).

\section{Conclusão}

A utilização do Hydropriming não foi vantajosa para promover a emergência e vigor nas sementes de Sesbania virgata, Cenostigma pyramidale, Vachelia farnesiana, Piptadenia retusa, Senna spectabilis, Mimosa tenuiflora, Sarcomphalus joazeiro e Enterolobium contortisiliquum. Outras pesquisas com o uso de técnicas de tratamento de sementes podem ser usadas a fim de melhorar o manejo destas espécies citadas.

Porém, a imersão por $24 \mathrm{~h}$ a $25^{\circ} \mathrm{C}$ pode ser potencial para promoção da emergência e vigor de Anadenanthera colubrina e Luetzelburgia auriculata, outras pesquisas podem ser realizadas para melhorar a utilização desta metodologia em virtude de um melhor manejo destas espécies.

\section{Agradecimentos}

Agradecemos ao Núcleo de Ecologia e Monitoramento Ambiental - NEMA/UNIVASF, o Projeto de Integração do Rio São Francisco com as Bacias Hidrográficas do Nordeste Setentrional - PISF e o Ministério do Desenvolvimento Regional - MDR pela disponibilização das sementes.

\section{Referências}

Abreu, D. C. A. D., Porto, K. G., \& Nogueira, A. C. (2017). Métodos de superação da dormência e substratos para germinação de sementes de Tachigali vulgaris L. G. Silva, \& H.C. Lima. Floresta e Ambiente, 24. https://doi.org/10.1590/2179-8087.071814.

Andrade, J. C., da Silva, A. R. P., Freitas, M. A., de Azevedo Ramos, B., Freitas, T. S., Franz de Assis, G., \& Coutinho, H. D. M. (2019). Control of bacterial and fungal biofilms by natural products of Ziziphus joazeiro Mart. (Rhamnaceae). Comparative immunology, microbiology and infectious diseases, 65, 226233.

Araujo, E. C. D., Mendonça, A. V. R., Barroso, D. G., Lamônica, K. R., \& Silva, R. F. D. (2004). Caracterização morfológica de frutos, sementes e plântulas de Sesbania virgata (Cav.) Pers. Revista Brasileira de Sementes, 26(1), 105-110. http://dx.doi.org/10.1590/S0101-31222004000100016.

Azeredo, G. A. D., Paula, R. C. D., Valeri, S. V., \& Moro, F. V. (2010). Superação de dormência de sementes de Piptadenia moniliformis Benth. Revista Brasileira de Sementes, 32(2), 49-58.

Azerêdo, G. A. D., Paula, R. C. D., \& Valeri, S. V. (2016). Germinação de sementes de Piptadenia moniliformis Benth. sob estresse hídrico. Ciência Florestal, 26(1), 193-202. https://doi.org/10.5902/1980509821112

Azeredo, G. A. D., Paula, R. C. D., Valeri, S. V., \& Moro, F. V. (2010). Superação de dormência de sementes de Piptadenia moniliformis Benth. Revista Brasileira de Sementes, 32(2), 49-58. https://doi.org/10.1590/S0101-31222010000200006.

Benedito, C. P., Ribeiro, M. C. C., Torres, S. B., Camacho, R. G. V., Soares, A. N. R., \& Guimarães, L. M. S. (2011). Armazenamento de sementes de catanduva (Piptadenia moniliformis Benth.) em diferentes ambientes e embalagens. Revista Brasileira de Sementes, 33(1), 28-37.

Bispo, G. L., Marco, C. A., da Silva Ferreira, F., Chaves, J. T. L., \& de Farias, R. B. (2019). Estudo etnobotânico de plantas medicinais no comércio da cidade de Juazeiro do Norte, CE. Journal of Biology \& Pharmacy and Agricultural Management, 15(4).

Brito, W. A. L., Pereira, K. T. O., Nogueira, N. W., Torres, S. B., \& de Paiva, E. P. (2020). Avaliação da viabilidade de sementes de Tabebuia aurea por meio do teste de tetrazólio. Revista Caatinga, 33(4), 993-999.

Castro, L. E., Guimarães, C. C., \& Faria, J. M. R. (2017). Physiological, cellular and molecular aspects of the desiccation tolerance in Anadenanthera colubrina seeds during germination. Brazilian Journal of Biology, 77(4), 774-780. 
Correia, L. A.S., de Medeiros, J. A. D., da Silva, A. B., dos Santos Ferrari, C., \& Pacheco, M. V. Qualidade fisiológica de sementes de catanduva sob infestação de Callosobruchus maculatus (Coleoptera: Bruchidae). Revista Agropecuária Técnica, 38(2).

Cruz, M. S. F. V., Ristau, A. C. P., de Matos Malavasi, M., \& Klosowski, É. S. (2020). Biometria e Potencial Fisiológico de Sementes de Anadenanthera colubrina (Vell.) Brenan em Função do Estádio de Desenvolvimento do Fruto. Cadernos de Agroecologia, 15(4).

Dantas, B. F., Ribeiro, R. C., Oliveira, G. M. D., Silva, F. F. S. D., \& Araújo, G. G. L. D. (2019). Biosaline production of seedlings of native species from the Caatinga dry forest. Ciência Florestal, 29(4), 1551-1567.

Dantas, B. F., Moura, M. S., Pelacani, C. R., Angelotti, F., Taura, T. A., Oliveira, G. M., \& Seal, C. E. (2020). Rainfall, not soil temperature, will limit the seed germination of dry forest species with climate change. Oecologia, 192(2), 529-541.

Freitas, T. A. S., Mendonça, A. V. R., do Nascimento, K. F., de Oliveira, L. F. B., \& Souza, L. S. (2019). Temperatura e fotoperíodo sobre a germinação de sementes de Poincianella pyramidalis (Tul.) LP Queiroz. MAGISTRA, 30, 94-103.

Bispo, J. S., da Costa, D. C. C., Gomes, S. E. V., de Oliveira, G. M., Matias, J. R., Ribeiro, R. C., \& Dantas, B. F. (2017). Size and vigor of Anadenanthera colubrina (Vell.) Brenan seeds harvested in Caatinga areas. Journal of Seed Science, 39(4), 363-373.

Du Bocage, A. L., \& Sales, M. F. D. (2002). The family Bombacaceae Kunth in the state of Pernambuco-Brazil. Acta Botanica Brasilica, 16(2), $123-139$.

Jeller, H., \& Perez, S. C. J. G. D. A. (2001). Efeitos dos estresses hídrico e salino e da ação de giberelina em sementes de Senna spectabilis. Ciência Florestal, 11(1), 93-104.

Lazarotto, M., Piveta, G., Muniz, M. F. B., \& Reiniger, L. R. S. (2011). Adequação do teste de tetrazólio para avaliação da qualidade de sementes de Ceiba speciosa. Semina: Ciências Agrárias, 32(4), 1243-1250.

Lazarotto, M., Muniz, M. F. B., \& Santos, Á. F. D. (2010). Detection, transmission, pathogenicity and chemical treatment of fungi in Ceiba speciosa seeds. Summa Phytopathologica, 36(2), 134-139.

Lima, A. T., \& Meiado, M. V. (2018). Effect of hydration and dehydration cycles on Mimosa tenuiflora seeds during germination and initial development. South African Journal of Botany, 116, 164-167.

Lima, A. T., Oliveira, D., \& Meiado, M. V. (2018). Effect of hydration and dehydration cycles on Macroptilium atropurpureum seeds germination under water deficit conditions. Comunicações em Plant Sciences, 8, 55-61.

Lima, A. T., Cunha, P. H. D. J. D., Dantas, B. F., \& Meiado, M. V. (2018). Does discontinuous hydration of Senna spectabilis (DC.) HS Irwin \& Barneby var. excelsa (Schrad.) HS Irwin \& Barneby (Fabaceae) seeds confer tolerance to water stress during seed germination?. Journal of Seed Science, $40(1), 36-43$.

Lima, A. T., \& Meiado, M. V. (2018). Effects of seed hydration memory on initial growth under water deficit of cactus from two populations that occur in different ecosystems in Northeast Brazil. Plant Species Biology, 33(4), 268-275.

Lopes, J. C., Matheus, M. T., Corrêa, N. B., \& da Silva, D. P. (2008). Germinação de sementes de embiruçu (Pseudobombax grandiflorum (Cav.) A. Robyns) em diferentes estádios de maturação e substratos. Floresta, 38(2).

Lorenzi, H. (2002) Árvores brasileiras: manual de identificação e cultivo de plantas arbóreas do Brasil. Instituto Plantarum, Nova Odessa.

Lozano, E. C., Zapater, M. A., Mamani, C., Flores, C. B., Gil, M. N., \& Sühring, S. S. (2016). Efecto de pretratamientos en semillas de Enterolobium contortisiliquum (Fabaceae) de la selva pedemontana argentina. Boletín de la Sociedad Argentina de Botánica, 51(1), 79-87.

Lucas, C. M., Mekdeçe, F., Nascimento, C. M., Holanda, A. S. S., Braga, J., Dias, S., \& Suemitsu, C. (2012). Effects of short-term and prolonged saturation on seed germination of Amazonian floodplain forest species. Aquatic Botany, 99, 49-5510.1016/j.aquabot.2012.02.004

Maciel, C. G., Bovolini, M. P., Finger, G., Pollet, C. S., \& Muniz, M. F. B. (2013). Avaliação de temperaturas e substratos na germinação de sementes de Jacaranda mimosifolia D. Don. Floresta e Ambiente, 20(1), 55-61.

Masetto, T. E., Faria, J. M. R., Fraiz, A. C. R., \& Rezende, R. K. S. (2013). Condicionamento osmótico de sementes de Sesbania virgata (CAV.) PERS (Fabaceae). Cerne, 19(4), 629-636. https://doi.org/10.1590/S0104-77602013000400013.

Matias, J. R., Torres, S. B., Freire, J. N. T., Alencar, S. D. S., \& Dantas, B. F. (2018). Germination of Cenostigma pyramidale seeds under different temperatures and salinities. Informativo ABRATES, 28(1), 115-118.

Matias, J. R., Torres, S. B., Freire, J. N. T., Alencar, S. D. S., \& Dantas, B. F. (2018). Germinação de sementes de Cenostigma pyramidale sob diferentes temperaturas e salinidades. In Embrapa Semiárido-Artigo em anais de congresso (ALICE). Informativo Abrates, 28(1).

Medeiros, J. X., Girlanio da Silva, U. F. C. G., Ramos, T., Lucena, D., \& Lúcio, A. (2013). Efeito de substratos na germinação de sementes de embiratanha (Pseudobombax marginatum) e métodos de superação de dormência em sementes de jucá (Caesalpinea ferrea). Engenharia Ambiental: Pesquisa e Tecnologia, 10(3).

Mesquita, A. C., Dantas, B. F., \& Cairo, P. A. R. (2018). Ecophysiology of caatinga native species under semi-arid conditions. Bioscience Journal, 34(6).

Moraes, F. G. D., Matos, V. P., Silva, M., Ferreira, E. G. B. S., Santos, H. H. D., Rodrigues, I. A. S., \& Bittar, S. M. B. (2012). Tratamentos pré-germinativos em sementes de Vachellia farnesiana (L.) Wight \& Arn.-Leguminosae-Mimosoidae. Scientia Plena, 8(4 (b)).

Nascimento, J. P. B., Meiado, M. V., \& Siqueira-Filho, J. A. (2018). Seed germination of three endangered subspecies of Discocactus Pfeiff. (Cactaceae) in response to environmental factors. Journal of Seed Science, 40(3), 253-262. 
Nascimento, J. P. B., \& Dantas, B. F. (2018) Angico-de-bezerro: Pityrocarpa moniliformis (Benth.) Luckow \& R.W. Jobson. NOTA TÉCNICA: ABRATES.

Nogueira, F. C. B., Silva, J. W. L., Bezerra, A. M. E., \& Medeiros Filho, S. (2012). Efeito da temperatura e luz na germinação de sementes de Luetzelburgia auriculata (Alemão) Ducke-Fabaceae. Acta Botanica Brasilica, 26(4), 772-778.

Oliveira, J. V., da Silva, M. X. G., Borges, A. K. M., Souto, W. M. S., de Faria Lopes, S., de Brito Melo Trovão, D. M., \& Alves, R. R. N. (2020). Fauna and conservation in the context of formal education: a study of urban and rural students in the semi-arid region of Brazil. Journal of ethnobiology and ethnomedicine, 16, 1-15.

Oliveira, J. R. D., Costa, C. A. S. D., Bezerra, A. M. E., Abud, H. F., \& Lucena, E. M. P. D. (2018). Characterization of seeds, seedlings and initial growth of Jacaranda mimosifolia D. Don.(Bignoniaceae). Revista Árvore, 42(4).

Oliveira, K. N., Espírito-Santo, M. M., Silva, J. O., \& Melo, G. A. (2012). Ontogenetic and temporal variations in herbivory and defense of Handroanthus spongiosus (Bignoniaceae) in a Brazilian tropical dry forest. Environmental entomology, 41(3), 541-550.

Oliveira, K. S. D., Oliveira, K. S. D., \& Aloufa, M. A. I. (2012). Influência de substratos na germinação de sementes de Anadenanthera colubrina (Vell.) Brenan em condições de casa de vegetação. Revista Árvore, 36(6), 1073-1078.

Pádua, G. V. G., Demartelaere, A. C. F., de Souza Medeiros, M. L., da Silva, J. N., Rodrigues, M. H. B. S., Preston, H. A. F., \& da Silva, R. M. (2020). Influência do extrato de Mimosa tenuiflora (Willd.) Poir sobre a qualidade fisiológica em sementes de Mimosa caesalpiniifolia (Benth). Brazilian Journal of Development, 6(11), 90475-90488. https://doi.org/10.34117/bjdv6n11-454

Pereira, K. T. O., Aquino, G. S. M., Alves, T. R. C., Benedito, C. P., \& Torres, S. B. (2015). Electrical conductivity test in Piptadenia moniliformis Benth. seeds. Journal of Seed Science, 37(4), 199-205. http://dx.doi.org/10.1590/2317-1545v37n4152357.

Pereira, M. S. (2011). Manual técnico: Conhecendo e produzindo sementes da Caatinga. Fortaleza: Associação Caatinga, 2011. 60p.

Pessoa, R. C., Matsumoto, S. N., Morais, O. M., Vale, R. S. D., \& Lima, J. M. (2010). Germinação e maturidade fisiológica de sementes de Piptadenia viridiflora (Kunth.) Benth relacionadas a estádios de frutificação e conservação pós-colheita. Revista Árvore, 34(4), 617-625. https://doi.org/10.1590/S010067622010000400006 .

Rego, S. S., Ferreira, M. M., Nogueira, A. C., \& Grossi, F. (2007). Influência de potenciais osmóticos na germinação de sementes de Anadenanthera colubrina (Veloso) Brenan (Angico-branco)-Mimosaceae. Revista Brasileira de Biociências, 5(S2), 549-551.

Rifna, E. J., Ramanan, K. R., \& Mahendran, R. (2019). Emerging technology applications for improving seed germination. Trends in Food Science \& Technology, 86, 95-108.

Rocha, A. P., Matos, V. P., Sena, L. H. D. M., Pacheco, M. V., \& Ferreira, R. L. C. (2018). Métodos para superação da dormência em sementes de Garcinia gardneriana (Planch. \& Triana) Zappi. Ciência Florestal, 28(2), 505-514.

Roveri Neto, A., \& Paula, R. C. D. (2017). Variabilidade entre árvores matrizes de Ceiba speciosa St. Hil para características de frutos e sementes. Revista Ciência Agronômica, 48(2), 318-327. https://doi.org/10.5935/1806-6690.20170037

Santana Santos, C., Trindade Lima, A., \& Vinicius Meiado, M. (2018). Efeito da hidratação descontínua em sementes de Mimosa tenuiflora (Willd.) Poir.(Fabaceae) submetidas a déficit hídrico durante a germinação. Informativo Abrates, 28(1).

Santo, F. D. S. D. E., Silva-Castro, M. M. D., \& Rapini, A. (2012). Two new species of Handroanthus Mattos (Bignoniaceae) from the state of Bahia, Brazil. Acta Botanica Brasilica, 26(3), 651-657. https://doi.org/10.1590/S0102-33062012000300014.

Scalon, S. de P. Q., Mussury, R. M., Wathier, F., Gomes, A. A., Silva, K. A., Pierezan, L., \& Scalon Filho, H. (2008). Armazenamento, germinação de sementes e crescimento inicial de mudas de em Enterolobium contortisiliquum (Vell.) Morong Acta Scientiarum. Biological Sciences, $27(2)$, 107-112. https://doi.org/10.4025/actascibiolsci.v27i2.1318

Silva, J. H. C. S., dos Santos, R. M., de Medeiros, M. D. G., da Silva, W. R., \& de Azeredo, G. A. (2019). Emergência de plântulas e vigor de sementes de Anadenanthera colubrina (Vell.) Brenan (Fabaceae) em diferentes substratos e profundidades de semeadura. Caderno Verde de Agroecologia $e$ Desenvolvimento Sustentável, 9(7), 7054.

Santos, C. A., da Silva, N. V., Walter, L. S., da Silva, E. C. A., \& Nogueira, R. J. M. C. (2016). Germinação de duas espécies da caatinga sob déficit hídrico e salinidade. Pesquisa Florestal Brasileira, 36(87), 219-224.

Lucena, R. F. P., de Medeiros, P. M., de Lima Araújo, E., Alves, A. G. C., \& de Albuquerque, U. P. (2012). The ecological apparency hypothesis and the importance of useful plants in rural communities from Northeastern Brazil: An assessment based on use value. Journal of Environmental Management, 96(1), $106-115$.

Silva, P. E. M., Santiago, E. F., de Menezes Daloso, D., Marques da Silva, E., \& Oliveira Silva, J. (2011). Quebra de dormência em sementes de Sesbania virgata (Cav.) Pers. Idesia (arica), 29(2), 39-45. http://dx.doi.org/10.4067/S0718-34292011000200005.

Trindade-Lessa, B. F., Nobre-de Almeida, J. P., Lobo-Pinheiro, C., Melo-Gomes, F., \& Medeiros-Filho, S. (2015). Germination and seedling growth of Enterolobium contortisiliquum as a function of seed weight and temperature and light conditions. Agrociencia, 49(3), 315-327.

Xue, F., Zhu, C., Liu, F., Wang, S., Liu, H., \& Li, C. (2018). Effects of high-intensity ultrasound treatment on functional properties of plum (Pruni domesticae semen) seed protein isolate. Journal of the Science of Food and Agriculture, 98(15), 5690-5699. 